\title{
Different curcumin forms selectively bind fibrillar amyloid beta in post mortem Alzheimer's disease brains: Implications for in-vivo diagnostics
}

\author{
Jurre den Haan ${ }^{1 *} \mathbb{D}$, Tjado H. J. Morrema ${ }^{2}$, Annemieke J. Rozemuller ${ }^{2}$, Femke H. Bouwman ${ }^{1}$ \\ and Jeroen J. M. Hoozemans²
}

\begin{abstract}
The combined fluorescent and $A \beta$-binding properties of the dietary spice curcumin could yield diagnostic purpose in the search for a non-invasive A $\beta$-biomarker for Alzheimer's disease (AD). However, evidence on the binding properties of curcumin, its conjugates and clinically used bio-available formulations to AD neuropathological hallmarks is scarce. We therefore assessed the binding properties of different curcumin forms to different neuropathological deposits in post-mortem brain tissue of cases with AD, other neurodegenerative diseases, and controls. Post mortem brain tissue was histochemically assessed for the binding of curcumin, its isoforms, conjugates and bio-available forms and compared to routinely used staining methods. For this study we included brains of early onset $A D$, late onset $A D$, primary age-related tauopathy (PART), cerebral amyloid angiopathy (CAA), frontotemporal lobar degeneration (FTLD) with tau or TAR DNA-binding protein 43 (TDP-43) inclusions, dementia with Lewy bodies (DLB), Parkinson's disease (PD) and control cases without brain pathology. We found that curcumin binds to fibrillar amyloid beta (A $\beta$ ) in plaques and CAA. It does not specifically bind to inclusions of protein aggregates in FTLD-tau cases, TDP-43, or Lewy bodies. Curcumin isoforms, conjugates and bio-available forms show affinity for the same $A \beta$ structures. Curcumin staining overlaps with immunohistochemical detection of $A \beta$ in fibrillar plaques and CAA, and to a lesser extent cored plaques. A weak staining of neurofibrillary tangles was observed, while other structures immunopositive for phosphorylated tau remained negative. In conclusion, curcumin, its isoforms, conjugates and bio-available forms selectively bind fibrillar A $\beta$ in plaques and CAA in post mortem $A D$ brain tissue. Curcumin, being a food additive with fluorescent properties, is therefore an interesting candidate for in-vivo diagnostics in AD, for example in retinal fluorescent imaging.
\end{abstract}

Keywords: Curcumin, Amyloid-beta, Alzheimer's disease, Cerebral amyloid angiopathy (CAA), Neurodegeneration, Biomarker, Immunohistochemistry

\section{Introduction}

Aggregation and extracellular deposition of the amyloidbeta $(A \beta)$ peptide in amyloid plaques is a pathological hallmark and an early event in the pathophysiology of Alzheimer's disease (AD) $[4,18]$. Currently AD diagnosis can be made with help of clinical criteria with the

\footnotetext{
* Correspondence: j.denhaan1@vumc.nl

'Department of Neurology, Amsterdam Neuroscience, VU University Medical Center Alzheimer Center, Mailbox 7057, 1007, MB, Amsterdam, the Netherlands

Full list of author information is available at the end of the article
}

support of measurements of $A \beta_{1-42}$ and tau ${ }_{-181}$ in cerebrospinal fluid (CSF), amyloid-positron emission tomography (PET), cortical atrophy on magnetic resonance imaging (MRI), or hypo-metabolism by fluorodeoxyglucose-PET (FDG-PET) [35]. As these are all either invasive or time consuming investigations, a patient friendly and repeatable amyloid or $A \beta$ biomarker is urgently needed for diagnosis as well as therapy monitoring in clinical trials [15]. Curcumin is a dietary spice that has been shown to have combined fluorescent and $\mathrm{A} \beta$-binding properties $[16,37,39]$. Therefore, curcumin has the potential to

(C) The Author(s). 2018 Open Access This article is distributed under the terms of the Creative Commons Attribution 4.0 International License (http://creativecommons.org/licenses/by/4.0/), which permits unrestricted use, distribution, and 
be used as a tool in a non-invasive $A \beta$-directed diagnostic assay for AD. A recently published paper by Koronyo et al. shows possible application of curcumin for in-vivo detection of retinal amyloid [22].

Curcumin is a polyphenolic compound derived from the root Curcuma longa or turmeric, traditionally used in Indian cuisine and Ayurvedic medicine. Turmeric naturally contains three curcuminoids namely; curcumin $(\approx 77 \%)$, demethoxycurcumin $(\mathrm{DMC})(\approx 17 \%)$ and bis-demethoxycurcumin $(B D M C)(\approx 3 \%)[10]$. Curcumin is fluorescent by nature [16, 37]. It has anti-carcinogenic, anti-inflammatory, anti-oxidant and anti-angiogenic properties and binds to $A \beta$ and interferes with $A \beta$ aggregation $[10,28]$. More specifically curcumin binds $A \beta$-oligomers and fibrils in vitro $[41,52]$, binds plaques in $\mathrm{APP}_{\mathrm{SWE}} /$ $\mathrm{PS}_{\triangle \mathrm{E} 9}, \mathrm{Tg} 2576$ and $5 \mathrm{xFAD}$ transgenic mice $[23,29,30,53]$ and binds $A \beta$-plaques in post mortem human $\mathrm{AD}$ brain tissue [23, 39, 49, 53]. Two reports also described moderate binding of curcumin to neurofibrillary tangles in AD brain tissue [36, 39].

In order to apply curcumin as a tool for in-vivo detection of $\mathrm{A} \beta$ its poor bio-availability and in-vivo metabolism should be considered. The majority (35-89\%) of the orally administered curcumin is present in the feces, as the intestinal mucosa and mucus form a physical barrier $[14,43]$. Secondly, curcumin that does reach the circulation undergoes phase 1 (reduction) and phase 2 metabolism (conjugation) in the liver. Reductases reduce curcumin to dihydrocurcumin, tetrahydrocurcumin, hexahydrocurcumin etc. [31, 32, 42]. In addition, curcumin is conjugated to sulfates and glucuronides [5, 17, 31, 32]. Therefore, the majority of circulating curcumin is conjugated. To overcome curcumin's poor bio-availability, nano-particulate drug delivery systems have been developed for oral administration including micelles, solid lipid nanoparticles and liposomes (Theracurmin $^{\text {Tw }}[20,40,44]$, Novasol $^{\text {Tx }}[7,21,45]$, Longvida $\left.^{\text {TM }}[11,22]\right)$.

So far, there are no reports on the $A \beta$-binding properties of conjugated curcumin or bio-available forms of curcumin. Insight into binding properties of circulating curcuminoids in the blood (e.g. DMC, BDMC, and curcumin conjugates) and specific bio-available formulations is needed for the design of biomarker assays using oral (bio-available) curcumin formulations. In this study, we assessed the binding properties of curcumin, its isoforms, its conjugates and clinically used bioavailable curcumin supplements to neuropathological hallmarks of $\mathrm{AD}$ and other neurodegenerative features in post mortem brain tissue.

\section{Materials and methods}

\section{Post mortem brain tissue}

Post mortem brain tissue was obtained from the Netherlands Brain Bank (NBB; Amsterdam, the
Netherlands). Prior to death, donors signed informed consent for brain autopsy and use of brain tissue and medical records for research purposes. For this study we selected 5 early onset $\mathrm{AD}$ (EOAD)-patients, 5 late onset $\mathrm{AD}(\mathrm{LOAD})$ patients, $5 \mathrm{AD}$ cases with capillary cerebral amyloid angiopathy (CAA type-1), 5 controls, 5 cases with primary age related tauopathy (PART), 3 frontotemporal lobar degeneration (FTLD) cases with tau pathology (FTLD-tau), 1 FTLD case with TDP-43 pathology (FTLD-TDP), 2 Parkinson's disease (PD) cases and 1 dementia with Lewy bodies (DLB) case who donated their brains to the NBB between 2000 and 2014. EOAD cases had a reported disease onset before 65 years of age (mean age of death 61.6 years), while LOAD cases had reported disease onset $>65$ years. Neuropathological diagnosis of $\mathrm{AD}$ was made following the NIA-AA criteria including Thal-staging for $A \beta$ stage, Braak-andBraak-staging for NFTs and CERAD-staging for neuritic plaques $[1,38,48]$. Pathological diagnosis of CAA-type 1, FTLD (tau and TDP-43), DLB and PD were made following Thal-, Mackenzie-, McKeith- and Braak-staging criteria respectively [2, 25-27, 33, 34, 47]. Two of the FTLD-Tau cases were P301L mutations (chromosome 17), while one was a sporadic Pick's disease case. The FTLD-TDP43 case was a progranulin mutation. See Table 1 for cohort characteristics.

\section{Immunohistochemistry (IHC)}

For each case we assessed formalin-fixed paraffin-embedded tissue sections ( $5 \mu \mathrm{m}$ thick) from mesencephalon, hippocampus, frontal or occipital lobes. Paraffinembedded tissue sections were deparaffinized and rehydrated using xylene, alcohol and phosphate-buffered saline (PBS; pH 7.4). Endogenous peroxidase activity was blocked by $0.3 \% \mathrm{H}_{2} \mathrm{O}_{2}$-treatment of the sections. Heat induced antigen retrieval was performed in $0.01 \mathrm{M}$ citrate buffer $\mathrm{pH} 6.0$ using an autoclave. Sections were incubated overnight with primary antibody and subsequently washed with PBS (see details in Table 2). Primary antibodies were detected using EnVision (Dako, Glostrup, Denmark) followed by washing in PBS. Antibody complex binding was visualized using 3,3'-diaminobenzidine (DAB) and sections were counterstained with hematoxylin. Stained sections were covered using Quick-D (Klinipath, Beek, the Netherlands).

\section{Thioflavin-S staining}

Thioflavin-S staining was performed by incubating deparaffinized and rehydrated sections with $1 \%$ thioflavin-S (Sigma-Aldrich) solution (demineralized water) for $1 \mathrm{~min}$, followed by rinsing off excess thioflavin-S using 70\% alcohol. Following staining slides were washed using PBS and covered using Tris-buffered saline (TBS)/glycerol mounting medium. 
Table 1 Cohort characteristics

\begin{tabular}{|c|c|c|c|c|c|c|}
\hline \# & PM delay (h:min) & Pathological diagnosis (mutation) & Sex & Age & Braak & Amyloid \\
\hline 1 & $6: 35$ & Control & $\mathrm{F}$ & 92 & III & 0 \\
\hline 2 & $7: 10$ & Control & $\mathrm{F}$ & 78 & । & A \\
\hline 3 & $4: 35$ & Control & $\mathrm{F}$ & 78 & $\|$ & A \\
\hline 4 & $7: 15$ & Control & M & 95 & $\|$ & B \\
\hline 5 & $5: 15$ & Control & M & 83 & I & A \\
\hline 6 & $5: 00$ & EOAD & M & 61 & $\mathrm{Vl}$ & C \\
\hline 7 & 5:05 & EOAD & M & 59 & $\mathrm{Vl}$ & C \\
\hline 8 & 4:40 & EOAD & M & 62 & V & $B$ \\
\hline 9 & $4: 45$ & EOAD & M & 64 & V & $C$ \\
\hline 10 & $5: 15$ & EOAD & M & 62 & Vl & $C$ \\
\hline 11 & $5: 30$ & LOAD & M & 88 & $\mathrm{Vl}$ & $C$ \\
\hline 12 & 7:00 & LOAD & $F$ & 92 & V & $C$ \\
\hline 13 & 4:40 & LOAD & $F$ & 89 & V & $C$ \\
\hline 14 & $6: 25$ & LOAD & $\mathrm{F}$ & 91 & IV & $C$ \\
\hline 15 & 3:05 & LOAD & M & 74 & VI & $C$ \\
\hline 16 & $6: 05$ & CAA type 1 & M & 68 & $\|$ & $C$ \\
\hline 17 & $4: 20$ & CAA type 1 & M & 81 & V & $C$ \\
\hline 18 & $4: 20$ & CAA type 1 & $F$ & 96 & V & $C$ \\
\hline 19 & $3: 25$ & CAA type 1 & M & 94 & V & $C$ \\
\hline 20 & $6: 00$ & CAA type 1 & $\mathrm{~F}$ & 75 & V & $C$ \\
\hline 21 & $5: 30$ & PART & $F$ & 81 & $\|$ & 0 \\
\hline 22 & $3: 52$ & PART & $F$ & 89 & III & 0 \\
\hline 23 & 5:00 & PART & $F$ & 87 & $\|$ & 0 \\
\hline 24 & $5: 50$ & PART & $\mathrm{F}$ & 93 & ॥ & 0 \\
\hline 25 & $6: 35$ & PART & $\mathrm{F}$ & 103 & IV & 0 \\
\hline 26 & $3: 35$ & FTLD-TDP (Progranulin) & $\mathrm{F}$ & 76 & n.a. & A \\
\hline 27 & $5: 23$ & FTLD-Tau (P301L) & M & 60 & n.a. & 0 \\
\hline 28 & $6: 25$ & FTLD-Tau (P301L) & M & 64 & n.a. & 0 \\
\hline 29 & $6: 15$ & FTLD-Tau (PiD) & M & 60 & n.a. & 0 \\
\hline 30 & $24: 00$ & PD & M & 57 & । & 0 \\
\hline 31 & $33: 00$ & $\mathrm{LBD} / \mathrm{AD}$ & $\mathrm{F}$ & 78 & $\mathrm{Vl}$ & B \\
\hline 32 & $14: 00$ & PD & M & 68 & । & 0 \\
\hline
\end{tabular}

Abbreviations: CAA type 1 capillary cerebral amyloid angiopathy type 1, EOAD early onset Alzheimer's disease, FTLD-tau frontotemporal lobar degeneration with tau pathology, FTLD-TDP-43 frontotemporal lobar degeneration with TDP-43 pathology, LOAD late onset Alzheimer's disease, n.a. not applicable, PART primary age-related tauopathy, PiD Pick's disease, PM delay post-mortem delay and PD Parkinson's disease

Table 2 Primary antibodies used for immunohistochemistry and fluorescent staining

\begin{tabular}{lll}
\hline Antibody & Source & Species \\
\hline IC-16 & Carsten Korth, Heinrich Heine University Dusseldorf, Düsseldorf, Germany [50] & Mouse \\
4G8 & Biolegend, San Diego, CA, USA & Mouse \\
AT8 & Invitrogen, Thermo Fisher Scientific, MA, USA & Mouse \\
PTDP-43 & Cosmo Bio, Tokyo, Japan & Mouse \\
LB-509 & Zymed, Thermo Fisher Scientific, MA, USA & Mouse \\
\hline
\end{tabular}




\section{Curcuminoid staining}

Curcuminoids, conjugates and bio-available forms of curcumin were dissolved in $0.5 \mathrm{M} \mathrm{NaOH}$ to a stock concentration of $20 \mathrm{mg} / \mathrm{ml}$ (see details in Table 3). Final concentrations of $0.2,0.1$ and $0.05 \mathrm{mg} / \mathrm{ml}$ were prepared by dilution in PBS. Deparaffinized and rehydrated sections were incubated with curcumin solutions for $10 \mathrm{~min}$, followed by washing in PBS. Stained sections were covered using TBS/glycerol mounting medium.

Combined curcumin staining and immunohistochemistry Sections were deparaffinized and rehydrated, followed by blocking of endogenous peroxidase and antigen retrieval as described above. Subsequently, curcumin staining was performed, sections were washed in PBS, and immunohistochemistry was performed using anti-phosphorylated tau (anti-pTau, AT8,1:800) and anti-A $\beta$ (IC-16, 1:400). Incubation was performed overnight, followed by washing in PBS. Secondary fluorescently labeled antibody (Goat Anti-mouse 594, Life Tech, 1:250) was incubated for $1.5 \mathrm{~h}$, followed by washing in PBS. Sections were counterstained with 4'6-diamidino-2-phenylindole (DAPI) and covered with TBS/Glycerol.

\section{Imaging}

IHC sections were imaged by an Olympus BX41 microscope using Leica Application Suite (LAS) AF lite software (Wetzlar, Germany). Fluorescently stained slides were imaged by a Leica DMi8 fluorescence microscope

Table 3 Curcuminoids, conjugates and bio-available forms of curcumin used for staining

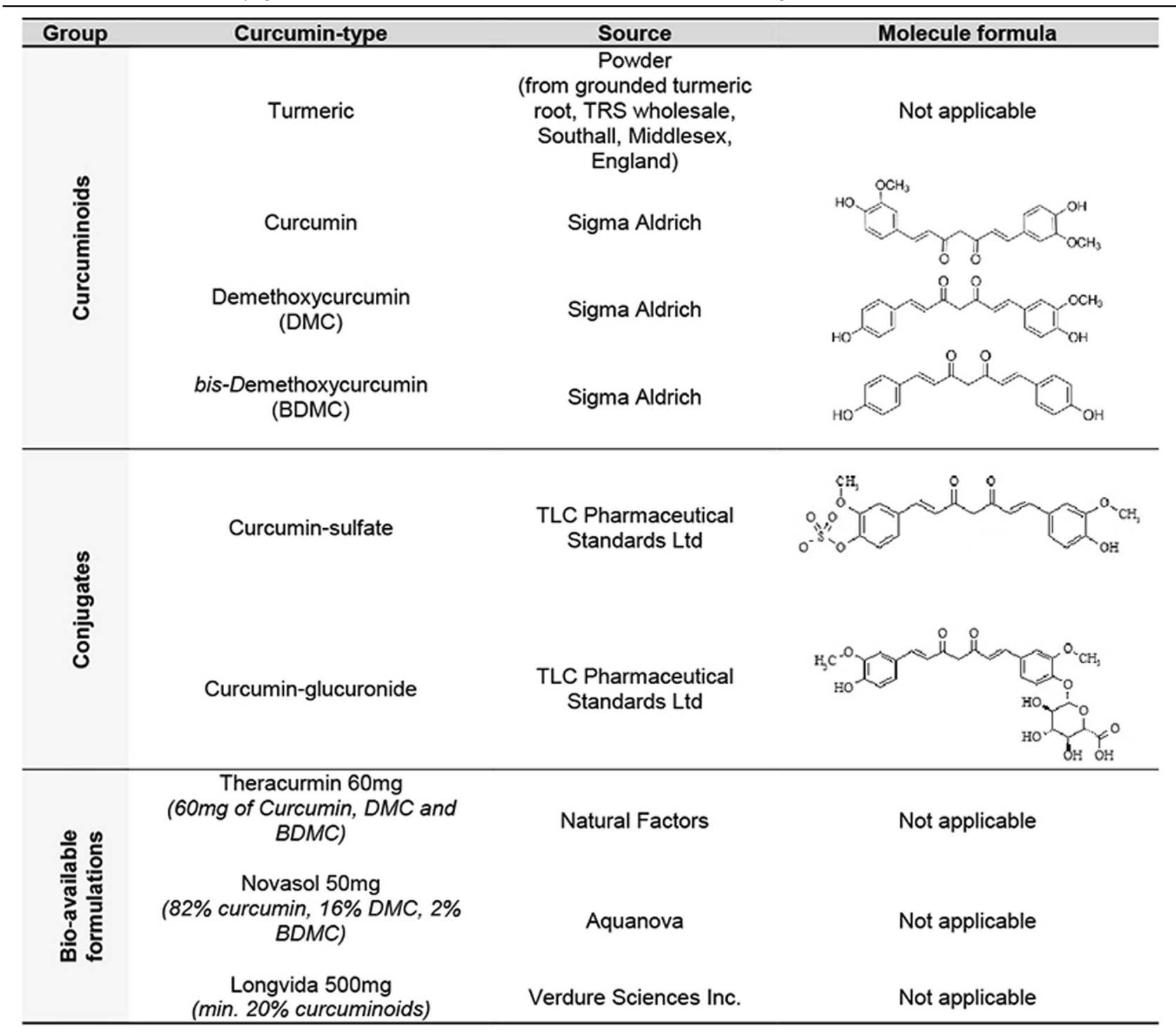


using LAS AF lite and ImageJ software (National Institue of Health, USA).

\section{Results}

\section{Curcumin binding in control and AD brain tissue}

In general, it was observed that curcumin binding on human brain tissue presented no background staining and auto-fluorescent signals in neurons. In brain tissue of EOAD, LOAD and AD cases with CAA (CAA type $1)$, curcumin strongly bound to amyloid plaques and CAA (Fig. 1). Curcumin staining revealed different types of plaques. We observed intense staining of primitive/ compact plaques and moderate staining of the cores in cored plaques. In $\mathrm{AD}$ cases, neurofibrillary tangles (NFTs) showed a weak detection by curcumin. Adjacent sections were assessed by immunohistochemistry for the presence of $\mathrm{A} \beta$ and $\mathrm{pTau}$ in a corresponding area, which confirmed the presence of these pathological structures.
The binding of curcumin to these pathological structures was investigated by co-stainings for curcumin and A $\beta$ (IC-16) or pTau (AT8). Primitive/compact plaques showed almost a complete overlap of curcumin signal and immunodetection by A $\beta$ (Fig. 2). Classic cored plaques presented co-labeling of the core, while the corona of these plaques were immuno-labelled by anti-A $\beta$ and not by curcumin. No binding of curcumin was observed in diffuse $A \beta$ deposits. CAA affected blood vessels were stained most intensively by curcumin and showed a complete overlap with the detection of CAA by A $\beta$. While compact plaques showed moderate neuritic changes, as presented by anti-pTau immuno-labeling, no co-labelling of anti-pTau and curcumin was present in these plaques (Fig. 3). Neuritic cored plaques showed a strong immunodetection of pTau around the core. The core of neuritic plaques showed a strong binding of curcumin while co-labelling with anti-pTau was absent. A strong

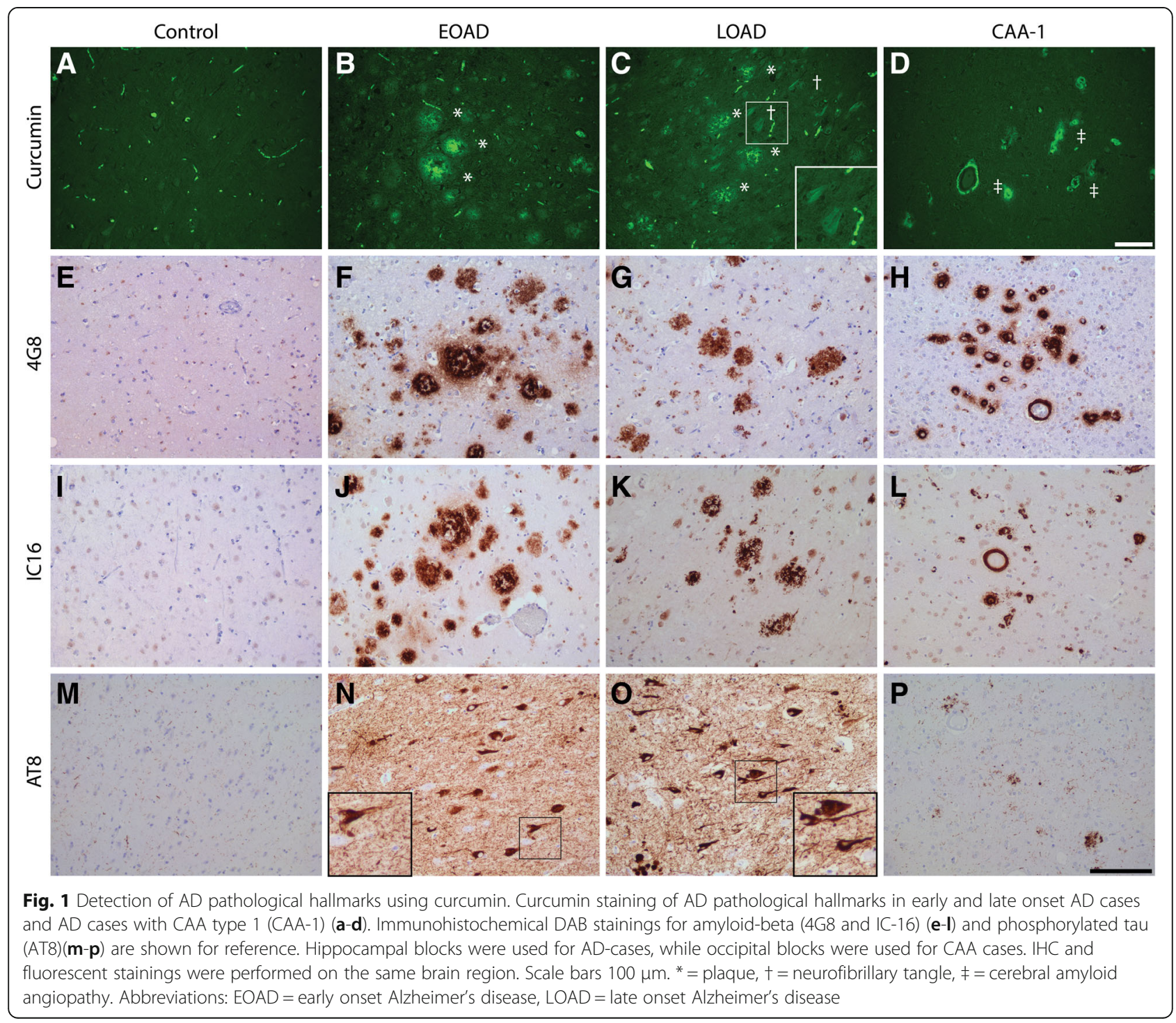




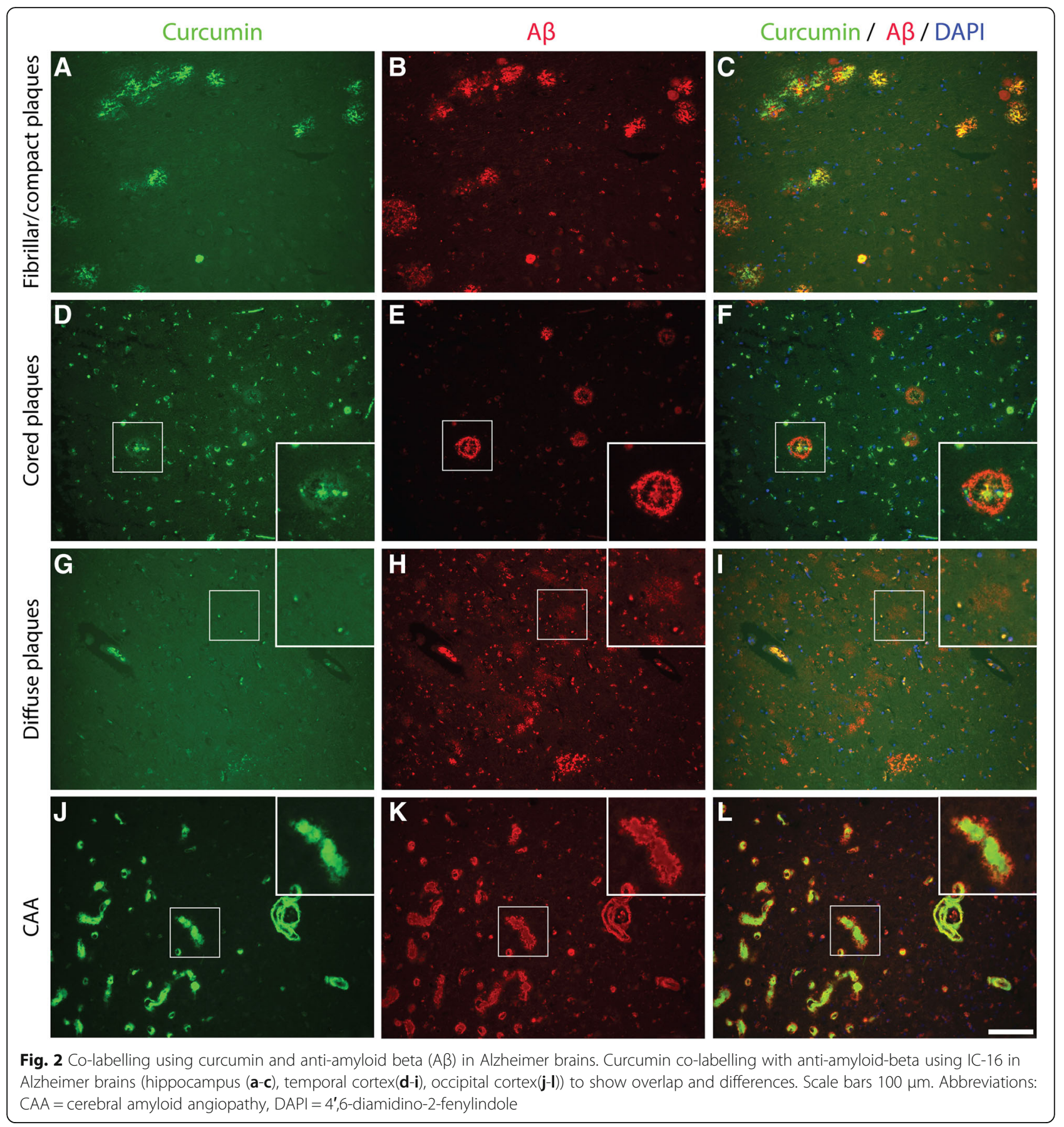

binding of curcumin was observed in CAA, however, no co-labelling with anti-pTau, observed around CAA affected capillaries, was detected. Co-labeling with antipTau confirmed that curcumin weakly detects NFTs.

\section{Curcumin does not bind to non-AD pathology}

We investigated whether curcumin showed binding to protein inclusions or aggregates in non-AD pathologies. PART is characterized by the presence of NFTs and tau pathology in the absence of amyloid deposits $[8,19]$. In cases with PART no specific binding of curcumin was observed. Co-labeling and single IHC with anti-pTau confirmed the presence of tau pathology and NFTs in the tissue section. In PART no binding of curcumin to NFTs was observed. Next, we assessed the binding of curcumin to tau inclusions in different FTLD-tau cases. Inclusions that were positive for pTau in cases with a MAPT P301L mutation, or in a case with sporadic 


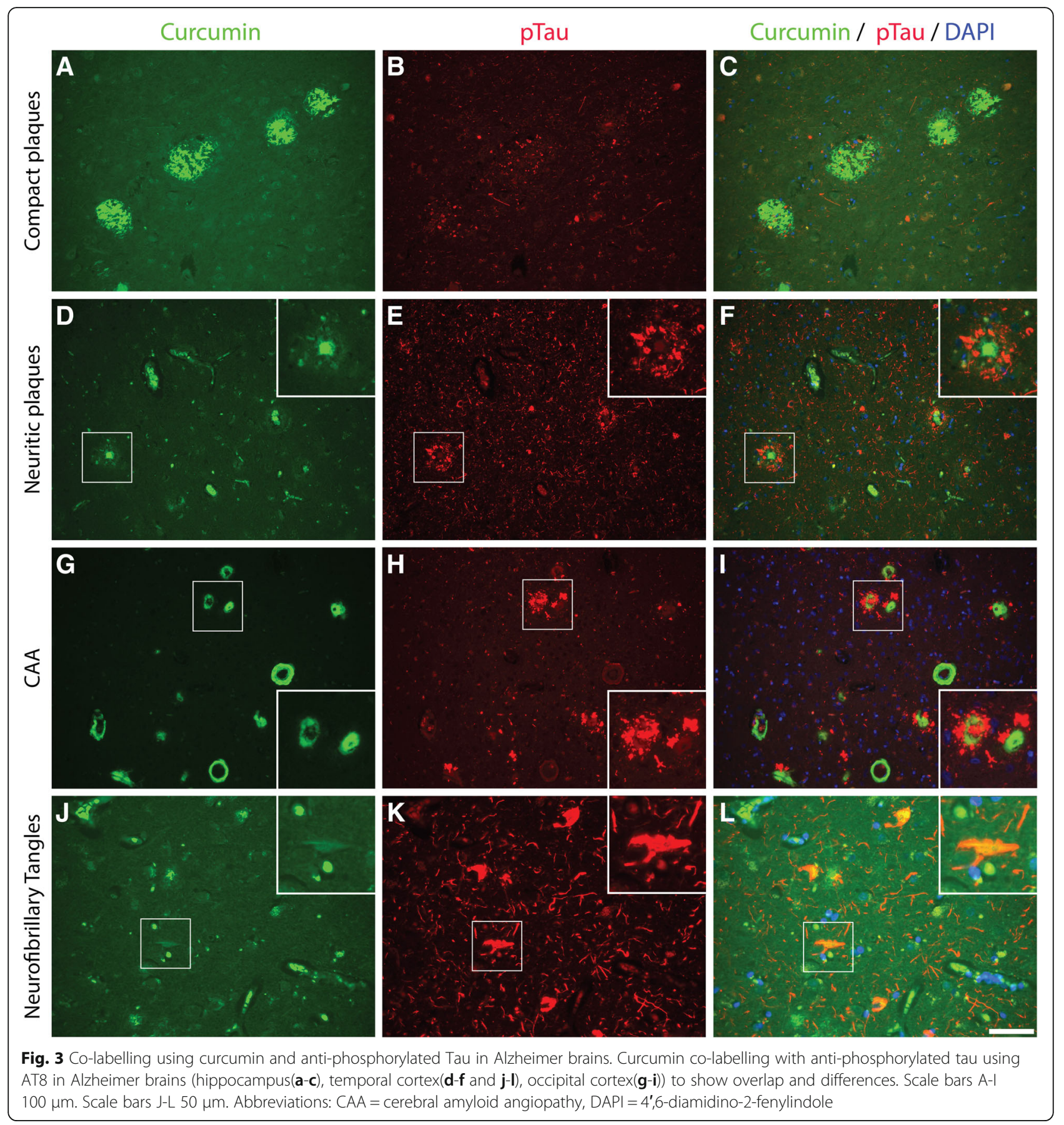

Pick's disease, did not show binding of curcumin. Also inclusions positive for TDP-43 in FTLD-TDP, and $\alpha$-synuclein containing inclusions in PD and DLB were not detected by curcumin (Fig. 4).

\section{Staining of curcumin isoforms, conjugates and bio-} available forms

In order to translate above findings to a possible in-vivo biomarker assay we assessed binding properties of different curcuminoids (DMC, BDMC), conjugates, and bio- available forms in AD cases. Given curcumin's chemical similarities with Thioflavin, sharing an aromatic ring, we compared stainings to Thioflavin-S staining (Fig. 5). An overview of staining of different curcumin forms and Thioflavin-S to plaques, CAA and NFTs is given in Table 4.

First, as turmeric also contains two isoforms: DMC $(\approx 17 \%)$ and BDMC $(\approx 3 \%)$, we assessed their binding. Similar to Thioflavin-S and curcumin, both isoforms bound amyloid plaques and CAA and showed weak 


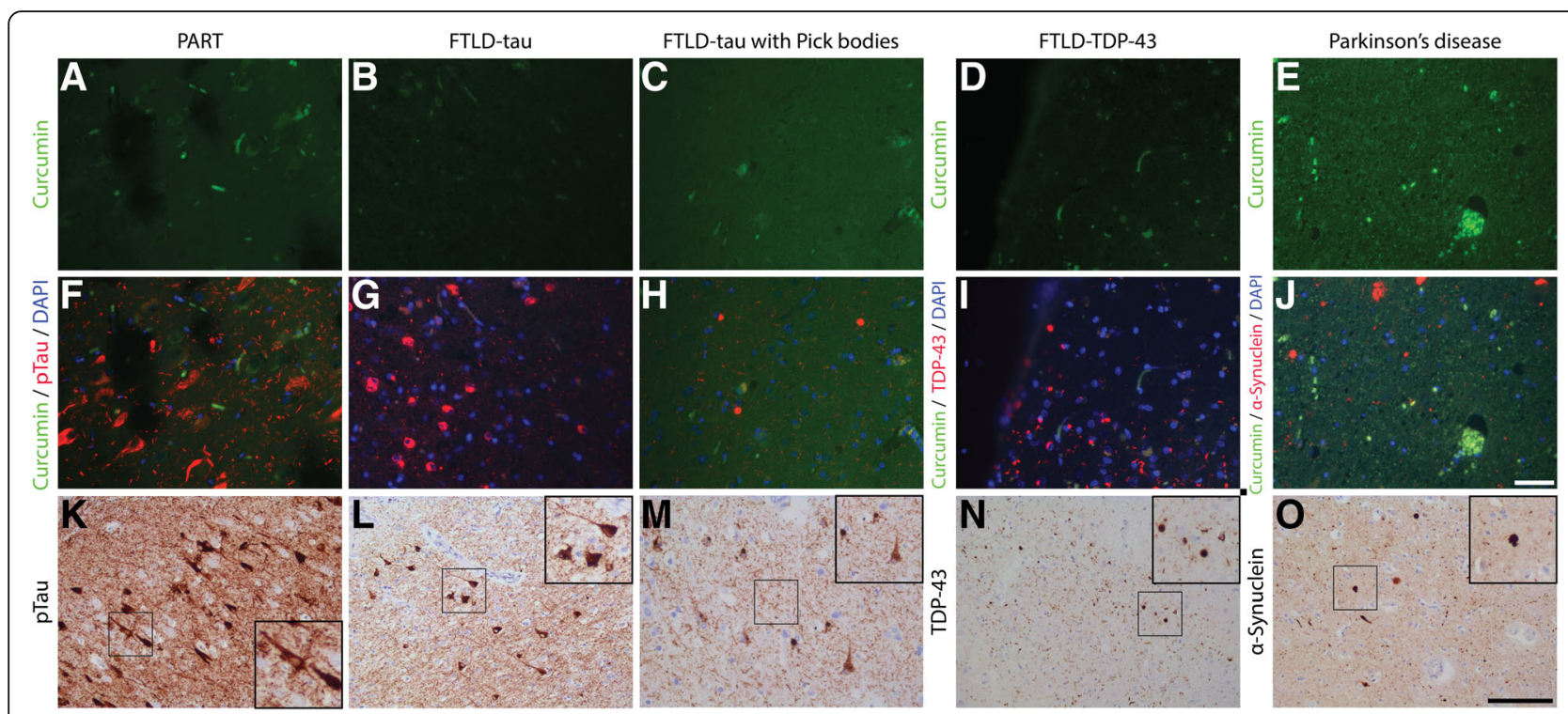

Fig. 4 Curcumin binding in non-AD pathologies. Curcumin staining of PART, FTLD-tau, FTLD-TDP-43, and PD cases (a-e). Fluorescent co-stainings for comparison of curcumin and tau (FTLD-tau), TDP-43 (FTD-TDP-43), and a-synuclein (PD) (f-j). Immunohistochemical DAB stainings for tau (AT8), TDP-43 (pTDP-43) and a-synuclein (LB-509) are shown for reference (k-o). Scale bars a-j 50 um. Scale bars k-o 100 um. Abbreviations: DAPI = 4,6-diamidino-2-fenylindole, FTLD-tau = frontotemporal lobe degeneration with tau pathology, FTLD-TDP-43= frontotemporal lobe degeneration with TDP-43 pathology, PART = primary age related tauopathy
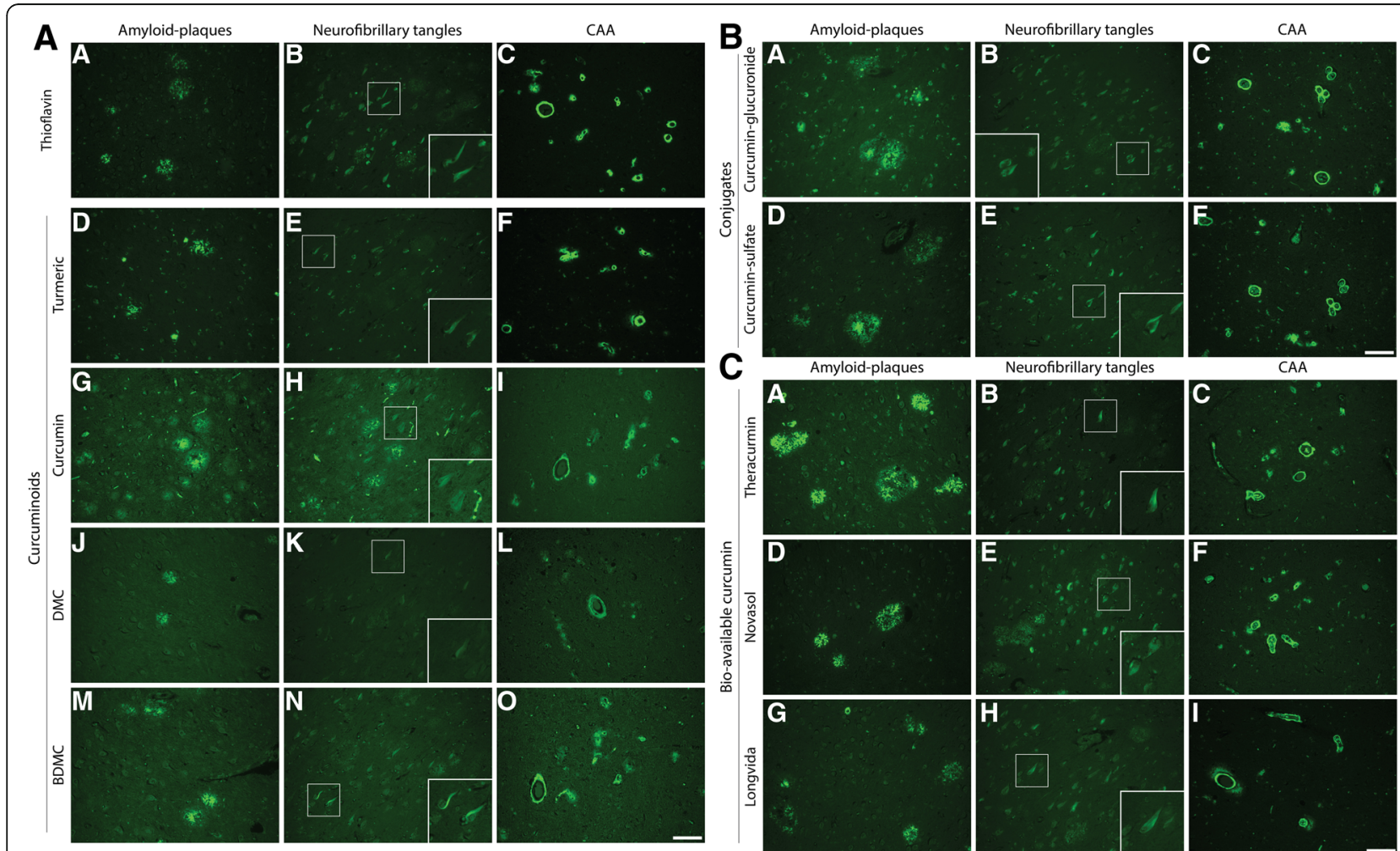

Fig. 5 Staining of different curcumin forms to amyloid plaques, neurofibrillary tangles and CAA. Staining of amyloid-beta plaques, neurofibrillary tangles and CAA with Thioflavin-S and curcuminoids (a), conjugates (b) and bio-available forms (c). Scale bars $100 \mu \mathrm{m}$ 
Table 4 Overview of binding of different curcumin forms to different pathological deposits

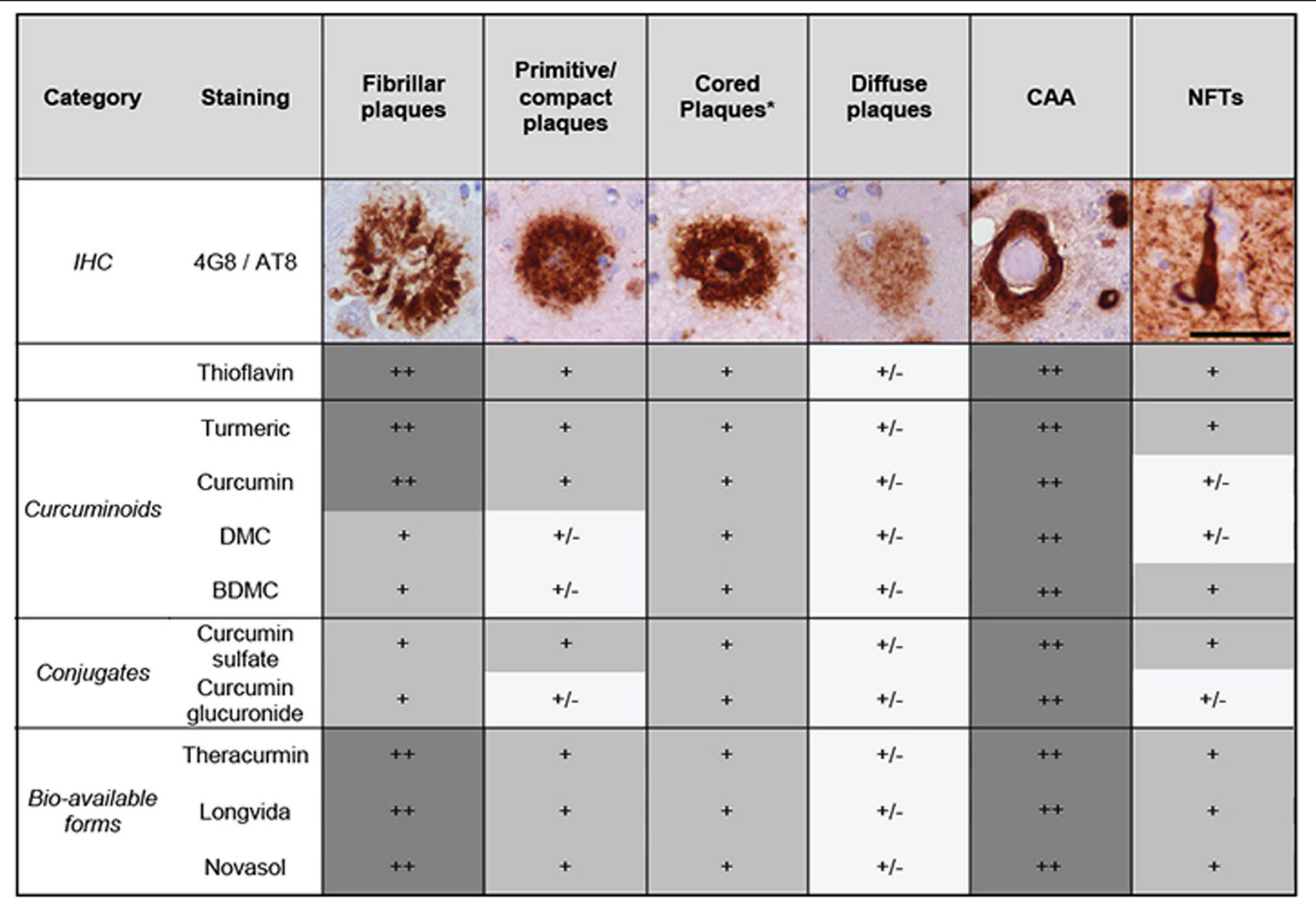

Staining of different pathological deposits by different curcumin forms was qualitatively compared for different plaque types, cerebral amyloid angiopathy (CAA) and neurofibrillary tangles (NFTs). Immuno-histochemical (IHC) stainings for amyloid-beta (4G8, plaques and CAA) and phosphorylated tau (AT-8, NFT's) are shown for reference. Scalebar $50 \mu \mathrm{m}$

++ : clearly visible, +: visible, +/-: visible with appreciation of morphology. ${ }^{*}$ The staining of the core of cored plaques rather than the corona was assessed here Abbreviations: $B D M C$ bis-demethoxycurcumin, DMC demethoxycurcumin

staining of NFTs (Fig. 5a). Compared to Thioflavin-S, staining of fibrillar and primitive plaques by isoforms was slightly less apparent (Table 4).

Next, we assessed binding properties of curcumin conjugates (glucuronides, sulfates). As, curcumin is conjugated by the liver to make it water soluble, conjugates represent the majority of circulating curcuminoids. Curcumin-glucuronide and curcumin-sulfate bound to amyloid plaques and CAA (Fig. 5b). Curcumin glucuronide showed slightly less apparent staining of fibrillar, primitive/compact plaques and NFTs compared to Thioflavin-S (Table 4).

Finally, in order to translate above findings to clinically used oral bio-available formulations of curcumin we compared three bio-available formulations, namely Theracurmin, Novasol and Longvida. All three forms showed staining of primitive/compact plaques, the core of cored plaques, CAA and faintly stain NFTs, similar to Thioflavin-S (Table 4, Fig. 5c).

\section{Discussion}

This study demonstrates that curcumin, curcuminoids, curcumin conjugates and bio-available formulations containing curcumin, bind to fibrillar amyloid in primitive/ compact plaques, cored plaques and $\mathrm{CAA}$ in $\mathrm{AD}$ brain tissue. Curcumin isoforms and conjugates show few selective binding to NFTs in AD brain tissue and no binding to other neuropathological deposits, e.g. pTau and TDP-43 positive structures in FTLD and alpha-synuclein inclusions in PD and DLB. To the best of our knowledge we are the first to report that curcumin isoforms, conjugates and bio-available forms have similar staining properties as curcumin.

Comparing plaque types, binding of curcumin was more pronounced to amyloid deposits with a dense amyloid structure, as shown in Fig. 2 and Table 4. This is supported by co detection of curcumin with amyloidbeta in compact plaques while in contrast, diffuse plaques showed a lack of co-detection (Fig. 3). This 
indicates that curcumin binds preferably to dense amyloid structures, such as fibrillar plaques, rather than the amyloid beta peptide itself. The detection of amyloid by curcumin is comparable to the detection of amyloid by Thioflavin-S. This could be explained by the fact that curcumin and Thioflavin have chemical similarities, sharing a double aromatic ring [52].

Previous studies showed that curcumin binds $\mathrm{A} \beta$-oligomers and fibrils in vitro [33, 44], $\mathrm{A} \beta$-plaques in APPSWE/ PS1 $\triangle \mathrm{E} 9, \mathrm{Tg} 2576$ and 5xFAD transgenic mice $[19,24,30,45]$ and in post mortem human AD brain tissue $[19,31,41,45]$. The latter human studies however assessed relatively small patient cohorts and did not assess other neurodegenerative diseases. Our findings in a well-characterized and large cohort of cases confirm earlier findings of specific binding of curcumin to fibrillar $A \beta$ plaques, weak binding to NFT's and rule out binding to pathological protein aggregates in other neurodegenerative diseases. Binding to fibrillary plaques might be of clinical relevance, as an increase of fibrillar plaques is associated with clinical progression to dementia [6]. In addition to curcumin binding to amyloid plaques, Mutsuga et al. described curcumin staining to CAA in larger vessels of an $\mathrm{AD}$ case as well as in various animals [39]. CAA can be subdivided in CAA type 1 (CAA-1) and CAA type 2(CAA-2) [47]. CAA-1 is characterized by $A \beta$ presence in leptomeningeal and cortical arteries, arterioles, veins and venules as well as cortical capillaries, while CAA-2 on the other hand, affects leptomeningeal and cortical vessels with the exception of capillaries. In addition to earlier findings in CAA-2 by Mutsuga et al. we showed that curcumin also binds to CAA in capillaries in CAA-1 cases [39].

To the existing literature we added the comparison of the binding properties of different curcumin forms in human brain tissue, which is needed to translate findings for possible use in in-vivo diagnostic or therapeutic studies. The majority of curcumin and its isoforms are conjugated in-vivo and conjugated curcumin represents a large proportion of circulating curcumin [12]. To overcome poor bio-availability, delivery systems containing curcuminoids were developed. We showed that isoforms and conjugates and newly developed delivery systems of curcumin bind amyloid plaques and CAA and show comparable fluorescent properties as curcumin. For diagnostic studies this implies that all contents of newly developed delivery systems, including curcumin, DMC an BDMC and secondary formed curcumin conjugates harbor amyloid binding properties and could yield diagnostic purpose. Newly developed delivery systems, e.g. micelles, solid lipid nanoparticles and liposomes, did not show to affect amyloid binding properties. Similar binding properties could also indicate a therapeutic role of isoforms and conjugates. Previous studies showed that curcumin reverses existing amyloid pathology and associated neurotoxicity in a mouse model $[9,53]$. If conjugates harbor the same functional effects on amyloid formation and related neurotoxicity is unknown. More research is therefore needed to assess possible functional effects of isoforms and conjugates on AD pathology.

Strengths of our study are the well-described patient cohort including various neurodegenerative features, thorough assessment of different curcumin forms and comparison with commonly used neuropathological IHC. Our patient cohort consists of a fair amount of well described AD-cases, cases with other neurodegenerative conditions as well as healthy controls, while previous studies assessed binding properties of curcumin mostly in animal models. Applying commonly used neuropathological IHC-staining we directly compared curcumin with established staining methods, which allows assessment of its specificity towards pathological structures. A limitation of our study is that the use of high concentrations curcumin in post mortem staining might not reflect concentrations that could be reached in-vivo. Although turmeric, with a mass percentage of only $\approx 3 \%$ curcuminoids, showed similar staining patterns and intensity as pure curcumin it should be noted that clinically reached concentrations might be unable to effectively bind pathology. Future studies might therefore test the minimal concentration needed to label pathology.

Secondly, we tested a wide variety of curcumin forms, however not synthesized forms of curcumin (e.g. CRANAD-28). CRANAD-28 might have amyloid plaques and CAA binding properties and beneficial blood brain barrier penetration, yet is not applicable for human use [54]. Lastly, as we focused on isoforms and conjugates representing the majority of circulating curcumin we did not test reduced forms of curcumin. Future studies might test binding properties of synthesized and reduced forms of curcumin.

The retina, as a protrusion of the central nervous system, might reflect neurodegenerative disease and is therefore of interest as a target for in-vivo fluorescent imaging both in ophthalmology and neurology. Previously, Cordeiro et al. visualized apoptotic cells in glaucoma patients with fluorescent imaging and showed a successful example of fluorescent molecular imaging in the retina [3]. Others claimed amyloid presence in both post mortem and in-vivo retinas of AD patients, visualized with curcumin [22-24]. This finding is controversial however, as other groups were unable to replicate post mortem detection of retinal amyloid [13, 46, 51]. Our results support the notion that, if fibrillar amyloid or amyloid angiopathy are present in the retina in $\mathrm{AD}$, 
curcuminoids might be used as labeling fluorophore for non-invasive fluorescent retinal imaging.

\section{Conclusion}

In conclusion, curcumin, its isoforms, conjugates and bio-available forms bind to fibrillar $A \beta$ plaques and CAA, and faintly stain neurofibrillary tangles in post mortem AD brain tissue. They do not show binding in control brain or to specific structures observed in other neurodegenerative disease like FTLD and PD. As conjugates and bio-available curcumin forms show comparable binding properties, curcumin might be an interesting candidate for in-vivo diagnostics in AD like retinal fluorescent imaging.

\section{Abbreviations}

AD: Alzheimer's disease; $A \beta$ : Amyloid-beta; BDMC: Bis-demethoxycurcumin; BMC: Demethoxycurcumin; CAA: Cerebral amyloid angiopathy; CAA1: Cerebral amyloid angiopathy type 1; CAA-2: Cerebral amyloid angiopathy type 2; CSF: Cerebrospinal fluid; DAB: 3,3' -diaminobenzidine; DAPI: 4'6diamidino-2-phenylindole; DLB: Dementia with Lewy bodies; EOAD: Early onset Alzheimer's disease; FTLD: Frontotemporal lobar degeneration; IHC: Immunohistochemistry; LOAD: Late onset Alzheimer's disease; MRI: Magnetic resonance imaging; NBB: The Netherlands Brain Bank; NFTs: Neurofibrillary tangles; PART: Primary age-related tauopathy; PBS: Phosphate-buffered saline; PD: Parkinson's disease; PET: Positron emission tomography; PiD: Pick's disease; PM delay: Post mortem delay; pTau: Phosphorylated tau; TBS: Tris-buffered saline; TDP-43: TAR DNA-binding protein 43

\section{Acknowledgements}

Anti-amyloid beta antibody (IC-16) was kindly provided by Carsten Korth, Heinrich Heine University, Dusseldorf, Germany.

\section{Funding}

This research was funded by Alzheimer Nederland.

\section{Authors' contributions}

JdH designed the study and experiments, performed analysis, prepared figures and drafted the manuscript. TM designed experiments, performed the experiments and performed analysis. JH performed analysis and supervised the study. FH designed and supervised the study. All authors read and approved the final manuscript.

\section{Ethics approval and consent to participate}

Prior to death, donors signed informed consent with the Netherlands Brain Bank (NBB) for brain autopsy and use of brain tissue and medical records for research purposes.

\section{Consent for publication}

Not applicable

\section{Competing interests}

The authors declare that they have no competing interests.

\section{Publisher's Note}

Springer Nature remains neutral with regard to jurisdictional claims in published maps and institutional affiliations.

\section{Author details}

'Department of Neurology, Amsterdam Neuroscience, VU University Medical Center Alzheimer Center, Mailbox 7057, 1007, MB, Amsterdam, the Netherlands. ${ }^{2}$ Department of Pathology, Amsterdam Neuroscience, VU University Medical Center, Amsterdam, the Netherlands.
Received: 21 June 2018 Accepted: 27 July 2018

Published online: 09 August 2018

\section{References}

1. Braak H, Alafuzoff I, Arzberger T, Kretzschmar H, Tredici K (2006) Staging of Alzheimer disease-associated neurofibrillary pathology using paraffin sections and immunocytochemistry. Acta Neuropathol 112:389-404. https:// doi.org/10.1007/s00401-006-0127-z

2. Braak H, Del Tredici K, Rub U, de Vos RA, Jansen Steur EN, Braak E (2003) Staging of brain pathology related to sporadic Parkinson's disease. Neurobiol Aging 24:197-211

3. Cordeiro MF, Normando EM, Cardoso MJ, Miodragovic S, Jeylani S, Davis BM, Guo L, Ourselin S, A'Hern R, Bloom PA (2017) Real-time imaging of single neuronal cell apoptosis in patients with glaucoma. Brain 140:17571767. https://doi.org/10.1093/brain/awx088

4. Cummings JL (2004) Alzheimer's disease. N Engl J Med 351:56-67. https:// doi.org/10.1056/NEJMra040223

5. Dhillon N, Aggarwal BB, Newman RA, Wolff RA, Kunnumakkara AB, Abbruzzese JL, Ng CS, Badmaev V, Kurzrock R (2008) Phase II trial of curcumin in patients with advanced pancreatic cancer. Clin Cancer Res 14 4491-4499. https://doi.org/10.1158/1078-0432.CCR-08-0024

6. Dickson TC, Vickers JC (2001) The morphological phenotype of betaamyloid plaques and associated neuritic changes in Alzheimer's disease. Neuroscience 105:99-107

7. Dutzmann S, Schiborr C, Kocher A, Pilatus U, Hattingen E, Weissenberger J, Gessler F, Quick-Weller J, Franz K, Seifert V et al (2016) Intratumoral concentrations and effects of orally administered micellar curcuminoids in glioblastoma patients. Nutr Cancer 68:943-948. https://doi.org/10.1080/ 01635581.2016 .1187281

8. Duyckaerts C, Braak H, Brion JP, Buee L, Del Tredici K, Goedert M, Halliday G, Neumann M, Spillantini MG, Tolnay M et al (2015) PART is part of Alzheimer disease. Acta Neuropathol 129:749-756. https://doi.org/10.1007/s00401-015-1390-7

9. Garcia-Alloza M, Borrelli LA, Rozkalne A, Hyman BT, Bacskai BJ (2007) Curcumin labels amyloid pathology in vivo, disrupts existing plaques, and partially restores distorted neurites in an Alzheimer mouse model. J Neurochem 102:1095-1104. https://doi.org/10.1111/j.1471-4159.2007.04613.x

10. Goel A, Kunnumakkara AB, Aggarwal BB (2008) Curcumin as "Curecumin": from kitchen to clinic. Biochem Pharmacol 75:787-809. https://doi.org/10. 1016/j.bcp.2007.08.016

11. Gota VS, Maru GB, Soni TG, Gandhi TR, Kochar N, Agarwal MG (2010) Safety and pharmacokinetics of a solid lipid curcumin particle formulation in osteosarcoma patients and healthy volunteers. J Agric Food Chem 58:20952099. https://doi.org/10.1021/jf9024807

12. Heger M, van Golen RF, Broekgaarden M, Michel MC (2014) The molecular basis for the pharmacokinetics and pharmacodynamics of curcumin and its metabolites in relation to cancer. Pharmacol Rev 66:222-307. https://doi. org/10.1124/pr.110.004044

13. Ho CY, Troncoso JC, Knox D, Stark W, Eberhart CG (2014) Beta-amyloid, phospho-tau and alpha-synuclein deposits similar to those in the brain are not identified in the eyes of Alzheimer's and Parkinson's disease patients. Brain Pathol 24:25-32. https://doi.org/10.1111/bpa.12070

14. Holder GM, Plummer JL, Ryan AJ (1978) The metabolism and excretion of curcumin (1,7-bis-(4-hydroxy-3-methoxyphenyl)-1,6-heptadiene-3,5-dione) in the rat. Xenobiotica 8:761-768

15. Hooper C, Lovestone S, Sainz-Fuertes R (2008) Alzheimer's disease, diagnosis and the need for biomarkers. Biomark Insights 3:317-323

16. Hun Bong P (2000) Spectral and Photophysical behaviors of curcumin and curcuminoids. Bull Korean Chem Soc 21(1):81-86

17. Ireson CR, Jones DJ, Orr S, Coughtrie MW, Boocock DJ, Williams ML, Farmer PB, Steward WP, Gescher AJ (2002) Metabolism of the cancer chemopreventive agent curcumin in human and rat intestine. Cancer Epidemiol Biomark Prev 11:105-111

18. Jack CR, Holtzman DM (2013) Biomarker modeling of alzheimer's disease. Neuron 80:1347-1358. https://doi.org/10.1016/j.neuron.2013.12.003

19. Jellinger KA, Alafuzoff I, Attems J, Beach TG, Cairns NJ, Crary JF, Dickson DW Hof PR, Hyman BT, Jack CR Jr et al (2015) PART, a distinct tauopathy, different from classical sporadic Alzheimer disease. Acta Neuropathol 129: 757-762. https://doi.org/10.1007/s00401-015-1407-2

20. Kanai M, Imaizumi A, Otsuka Y, Sasaki H, Hashiguchi M, Tsujiko K, Matsumoto S, Ishiguro H, Chiba T (2012) Dose-escalation and pharmacokinetic study of nanoparticle curcumin, a potential anticancer 
agent with improved bioavailability, in healthy human volunteers. Cancer Chemother Pharmacol 69:65-70. https://doi.org/10.1007/s00280-011-1673-1

21. Kocher A, Bohnert L, Schiborr C, Frank J (2016) Highly bioavailable micellar curcuminoids accumulate in blood, are safe and do not reduce blood lipids and inflammation markers in moderately hyperlipidaemic individuals. Mol Nutr Food Res. https://doi.org/10.1002/mnfr.201501034

22. Koronyo Y, Biggs D, Barron E, Boyer DS, Pearlman JA, Au WJ, Kile SJ, Blanco A, Fuchs DT, Ashfaq Aet al (2017) Retinal amyloid pathology and proof-ofconcept imaging trial in Alzheimer's disease. JCI Insight 2: Doi https://doi. org/10.1172/jci.insight.93621

23. Koronyo-Hamaoui M, Koronyo Y, Ljubimov AV, Ca M, Ko MK, Black KL, Schwartz M, Farkas DL (2011) Identification of amyloid plaques in retinas from Alzheimer's patients and noninvasive in vivo optical imaging of retinal plaques in a mouse model. Neurolmage 54:S204-S217. https://doi.org/10. 1016/j.neuroimage.2010.06.020

24. La Morgia C, Ross-Cisneros FN, Koronyo Y, Hannibal J, Gallassi R, Cantalupo G, Sambati L, Pan BX, Tozer KR, Barboni P et al (2015) Melanopsin retinal ganglion cell loss in Alzheimer's disease. Ann Neurol. https://doi.org/10. 1002/ana.24548

25. Love S, Chalmers K, Ince P, Esiri M, Attems J, Jellinger K, Yamada M, McCarron M, Minett T, Matthews F et al (2014) Development, appraisal, validation and implementation of a consensus protocol for the assessment of cerebral amyloid angiopathy in post-mortem brain tissue. Am J Neurodegenerative Dis 3:19-32

26. Mackenzie IR, Neumann M, Bigio EH, Cairns NJ, Alafuzoff I, Kril J, Kovacs GG, Ghetti B, Halliday G, Holm IE et al (2010) Nomenclature and nosology for neuropathologic subtypes of frontotemporal lobar degeneration: an update. Acta Neuropathol 119:1-4. https://doi.org/10.1007/s00401-009-0612-2

27. Mackenzie IRA, Neumann M, Bigio EH, Cairns NJ, Alafuzoff I, Kril J, Kovacs GG, Ghetti B, Halliday G, Holm IE et al (2009) Nomenclature for neuropathologic subtypes of frontotemporal lobar degeneration: consensus recommendations. Acta Neuropathol 117:15-18. https://doi.org/10.1007/ s00401-008-0460-5

28. Maiti P, Dunbar GL (2018) Use of curcumin, a natural polyphenol for targeting molecular pathways in treating age-related neurodegenerative diseases. Int J Mol Sci 19. https://doi.org/10.3390/ijms19061637

29. Maiti P, Hall TC, Paladugu L, Kolli N, Learman C, Rossignol J, Dunbar GL (2016) A comparative study of dietary curcumin, nanocurcumin, and other classical amyloid-binding dyes for labeling and imaging of amyloid plaques in brain tissue of 5x-familial Alzheimer's disease mice. Histochem Cell Biol 146:609-625. https://doi.org/10.1007/s00418-016-1464-1

30. Maiti P, Paladugu L, Dunbar GL (2018) Solid lipid curcumin particles provide greater anti-amyloid, anti-inflammatory and neuroprotective effects than curcumin in the 5xFAD mouse model of Alzheimer's disease. BMC Neurosci 19:7. https://doi.org/10.1186/s12868-018-0406-3

31. Marczylo TH, Steward WP, Gescher AJ (2009) Rapid analysis of curcumin and curcumin metabolites in rat biomatrices using a novel ultraperformance liquid chromatography (UPLC) method. J Agric Food Chem 57:797-803. https://doi.org/10.1021/jf803038f

32. Marczylo TH, Verschoyle RD, Cooke DN, Morazzoni P, Steward WP, Gescher AJ (2007) Comparison of systemic availability of curcumin with that of curcumin formulated with phosphatidylcholine. Cancer Chemother Pharmacol 60:171-177. https://doi.org/10.1007/s00280-006-0355-x

33. McKeith IG, Boeve BF, Dickson DW, Halliday G, Taylor JP, Weintraub D, Aarsland D, Galvin J, Attems J, Ballard CG et al (2017) Diagnosis and management of dementia with Lewy bodies: fourth consensus report of the DLB consortium. Neurology 89:88-100. https://doi.org/10.1212/wnl. 0000000000004058

34. McKeith IG, Galasko D, Kosaka K, Perry EK, Dickson DW, Hansen LA, Salmon DP, Lowe J, Mirra SS, Byrne EJ et al (1996) Consensus guidelines for the clinical and pathologic diagnosis of dementia with Lewy bodies (DLB): report of the consortium on DLB international workshop. Neurology 47: 1113-1124

35. McKhann GM, Knopman DS, Chertkow H, Hyman BT, Jack CR, Kawas CH, Klunk WE, Koroshetz WJ, Manly JJ, Mayeux R et al (2011) The diagnosis of dementia due to Alzheimer's disease: recommendations from the National Institute on Aging-Alzheimer's Association workgroups on diagnostic guidelines for Alzheimer's disease. Alzheimers Dement 7:263-269. https:// doi.org/10.1016/j.jalz.2011.03.005

36. Mohorko N, Repovs G, Popovic M, Kovacs GG, Bresjanac M (2010) Curcumin labeling of neuronal fibrillar tau inclusions in human brain samples. J
Neuropathol Exp Neurol 69:405-414. https://doi.org/10.1097/NEN 0b013e3181d709eb

37. Mondal S, Ghosh S, Moulik SP (2016) Stability of curcumin in different solvent and solution media: UV-visible and steady-state fluorescence spectral study. J Photochem Photobiol B 158:212-218. https://doi.org/10. 1016/j.jphotobiol.2016.03.004

38. Montine TJ, Phelps CH, Beach TG, Bigio EH, Cairns NJ, Dickson DW, Duyckaerts C, Frosch MP, Masliah E, Mirra SS et al (2012) National Institute on Aging-Alzheimer's Association guidelines for the neuropathologic assessment of Alzheimer's disease: a practical approach. Acta Neuropathol 123:1-11. https://doi.org/10.1007/s00401-011-0910-3

39. Mutsuga M, Chambers JK, Uchida K, Tei M, Makibuchi T, Mizorogi T, Takashima A, Nakayama H (2012) Binding of curcumin to senile plaques and cerebral amyloid Angiopathy in the aged brain of various animals and to neurofibrillary tangles in Alzheimer's brain. J Vet Med Sci 74:51-57. https:// doi.org/10.1292/jvms.11-0307

40. Nakagawa Y, Mukai S, Yamada S, Matsuoka M, Tarumi E, Hashimoto T, Tamura C, Imaizumi A, Nishihira J, Nakamura T (2014) Short-term effects of highly-bioavailable curcumin for treating knee osteoarthritis: a randomized, double-blind, placebo-controlled prospective study. J Orthop Sci 19:933939. https://doi.org/10.1007/s00776-014-0633-0

41. Ono K, Hasegawa K, Naiki H, Yamada M (2004) Curcumin has potent antiamyloidogenic effects for Alzheimer's beta-amyloid fibrils in vitro. J Neurosci Res 75:742-750. https://doi.org/10.1002/jnr.20025

42. Pan MH, Huang TM, Lin JK (1999) Biotransformation of curcumin through reduction and glucuronidation in mice. Drug Metab Dispos 27:486-494

43. Ravindranath V, Chandrasekhara N (1981) Metabolism of curcumin--studies with [3H]curcumin. Toxicology 22:337-344

44. Sasaki H, Sunagawa Y, Takahashi K, Imaizumi A, Fukuda H, Hashimoto T, Wada H, Katanasaka Y, Kakeya H, Fujita M et al (2011) Innovative preparation of curcumin for improved oral bioavailability. Biol Pharm Bull 34:660-665. https://doi.org/10.1248/bpb.34.660

45. Schiborr C, Kocher A, Behnam D, Jandasek J, Toelstede S, Frank J (2014) The oral bioavailability of curcumin from micronized powder and liquid micelles is significantly increased in healthy humans and differs between sexes. Mol Nutr Food Res 58:516-527. https://doi.org/10.1002/mnfr.201300724

46. Schön C, Hoffmann NA, Ochs SM, Burgold S, Filser S, Steinbach S, Seeliger MW, Arzberger T, Goedert M, Kretzschmar HA et al (2012) Long-term in vivo imaging of Fibrillar tau in the retina of P301S transgenic mice. PLoS One 7: 1-9. https://doi.org/10.1371/journal.pone.0053547

47. Thal DR, Ghebremedhin E, Rub U, Yamaguchi H, Del Tredici K, Braak H (2002) Two types of sporadic cerebral amyloid angiopathy. J Neuropathol Exp Neurol 61:282-293

48. Thal DR, Rub U, Orantes M, Braak H (2002) Phases of a beta-deposition in the human brain and its relevance for the development of AD. Neurology 58:1791-1800

49. Veldman ER, Jia Z, Halldin C, Svedberg MM (2016) Amyloid binding properties of curcumin analogues in Alzheimer's disease postmortem brain tissue. Neurosci Lett 630:183-188. https://doi.org/10.1016/j.neulet.2016.07.045

50. Verwey NA, Hoozemans JJ, Korth C, van Royen MR, Prikulis I, Wouters D, Twaalfhoven HA, van Haastert ES, Schenk D, Scheltens P et al (2013) Immunohistochemical characterization of novel monoclonal antibodies against the N-terminus of amyloid beta-peptide. Amyloid 20:179-187. https://doi.org/10.3109/13506129.2013.797389

51. Williams EA, McGuone D, Frosch MP, Hyman BT, Laver N, StemmerRachamimov A (2017) Absence of Alzheimer disease Neuropathologic changes in eyes of subjects with Alzheimer disease. J Neuropathol Exp Neurol 76:376-383. https://doi.org/10.1093/jnen/nl×020

52. Yanagisawa D, Taguchi H, Yamamoto A, Shirai N, Hirao K, Tooyama I (2011) Curcuminoid binds to amyloid-beta1-42 oligomer and fibril. J Alzheimers Dis 24(Suppl 2):33-42. https://doi.org/10.3233/JAD-2011-102100

53. Yang F, Lim GP, Begum AN, Ubeda OJ, Simmons MR, Ambegaokar SS, Chen PP, Kayed R, Glabe CG, Frautschy SA et al (2005) Curcumin inhibits formation of amyloid beta oligomers and fibrils, binds plaques, and reduces amyloid in vivo. J Biol Chem 280:5892-5901. https://doi.org/10.1074/jbc.M404751200

54. Zhang X, Tian Y, Yuan P, Li Y, Yaseen MA, Grutzendler J, Moore A, Ran C (2014) A bifunctional curcumin analogue for two-photon imaging and inhibiting crosslinking of amyloid beta in Alzheimer's disease. Chem Commun (Camb) 50:11550-11553. https://doi.org/10.1039/c4cc03731f 